\title{
Upper and lower solution method for $n$ th-order BVPs on an infinite interval
}

\author{
Hairong Lian ${ }^{1 *}$, Junfang Zhao ${ }^{1}$ and Ravi P Agarwal ${ }^{2}$
}

"Correspondence: lianhr@126.com ${ }^{1}$ School of Science, China University of Geosciences, Beijing, 100083 , PR China

Full list of author information is available at the end of the article

\begin{abstract}
This work is devoted to the study of $n$ th-order ordinary differential equations on a half-line with Sturm-Liouville boundary conditions. The existence results of a solution, and triple solutions, are established by employing a generalized version of the upper and lower solution method, the Schäuder fixed point theorem, and topological degree theory. In our problem the nonlinearity depends on derivatives, and we allow solutions to be unbounded, which is an extra interesting feature.
\end{abstract}

MSC: $34 \mathrm{~B} 15 ; 34 \mathrm{~B} 40$

Keywords: higher order; boundary value problem; half-line; upper solution; lower solution

\section{Introduction}

In this paper, we study $n$ th-order ordinary differential equations on a half-line,

$$
-u^{(n)}(t)=q(t) f\left(t, u(t), \ldots, u^{(n-1)}(t)\right), \quad 0<t<+\infty,
$$

together with the Sturm-Liouville boundary conditions

$$
\left\{\begin{array}{l}
u^{(i)}(0)=A_{i}, \quad i=0,1, \ldots, n-3 \\
u^{(n-2)}(0)-a u^{(n-1)}(0)=B \\
u^{(n-1)}(+\infty)=C
\end{array}\right.
$$

where $q:(0,+\infty) \rightarrow(0,+\infty), f:[0,+\infty) \times \mathbb{R}^{n} \rightarrow \mathbb{R}$ are continuous, $a>0, A_{i}, B, C \in \mathbb{R}$, $i=0,1, \ldots, n-3, u(+\infty)=\lim _{t \rightarrow+\infty} u(t)$.

Higher-order boundary value problems (BVPs) have been studied in many papers, such as [1-3] for two-point BVP, [4, 5] for multipoint BVP, and [6-9] for infinite interval problem. However, most of these works have been done either on finite intervals, or for bounded solutions on an infinite interval. The authors in $[1,2,5,10-15]$ assumed one pair of well-ordered upper and lower solutions, and then applied some fixed point theorems or a monotone iterative technique to obtain a solution. In $[5,11,16]$, the authors assumed two pairs of upper and lower solutions and showed the existence of three solutions.

Infinite interval problems occur in the study of radially symmetric solutions of nonlinear elliptic equations; see $[6,7,17]$. A principal source of such problems is fluid dynamics. In boundary layer theory, Blasius-type equations lead to infinite interval problems. Semiconductor circuits and soil mechanics are other applied fields. In addition, some singular

\section{Springer}

@2014 Lian et al.; licensee Springer. This is an Open Access article distributed under the terms of the Creative Commons Attribution License (http://creativecommons.org/licenses/by/2.0), which permits unrestricted use, distribution, and reproduction in any medium, provided the original work is properly cited. 
boundary value problems on finite intervals can be converted into equivalent nonlinear problems on semi-infinite intervals [7]. During the last few years, fixed point theorems, shooting methods, upper and lower technique, etc. have been used to prove the existence of a single solution or multiple solutions to infinite interval problems; see [6-13, 17-24] and the references therein.

When applying the upper and lower solution method to infinite interval problems, the solutions are always assumed to be bounded. For example, in [10], Agarwal and O'Regan discussed the following second-order Sturm-Liouville boundary value problem:

$$
\left\{\begin{array}{l}
\frac{1}{p(t)}\left(p(t) y^{\prime}(t)\right)^{\prime}=q(t) f(t, y(t)), \quad t \in(0,+\infty), \\
-a_{0} y(0)+b_{0} \lim _{t \rightarrow 0^{+}} p(t) y^{\prime}(t)=c_{0}, \quad \text { or } \quad \lim _{t \rightarrow 0^{+}} p(t) y^{\prime}(t)=0, \\
y(t) \text { bounded on }[0,+\infty), \quad \text { or } \quad \lim _{t \rightarrow+\infty} y(t)=0,
\end{array}\right.
$$

where $a_{0}>0, b_{0} \geq 0$. They established existence criteria by using a diagonalization argument and existence results of appropriate boundary value problems on finite intervals.

Eloe et al. [11] studied the BVP

$$
\left\{\begin{array}{l}
x^{\prime \prime}(t)-a(t) x(t)+f(t, x(t))=0, \quad t \in(0,+\infty) \\
x(0)=x_{0}, \quad x(t) \text { bounded on }[0,+\infty)
\end{array}\right.
$$

They employed the technique of lower and upper solutions and the theory of fixed point index to obtain the existence of at least three solutions.

The problems related to global solutions, especially when the boundary data are prescribed asymptotically and the solutions may be unbounded, have been briefly discussed in [7, 9]. Recently, Yan et al. [14] developed the upper and lower solution theory for the boundary value problem

$$
\left\{\begin{array}{l}
y^{\prime \prime}(t)+\Phi(t) f\left(t, y(t), y^{\prime}(t)\right)=0, \quad t \in(0,+\infty), \\
a y(0)-b y^{\prime}(0)=y_{0} \geq 0, \quad \lim _{t \rightarrow+\infty} y^{\prime}(t)=k>0,
\end{array}\right.
$$

where $a>0, b>0$. By using the upper and lower solutions method and a fixed point theorem, they presented sufficient conditions for the existence of unbounded positive solutions; however, their results are suitable only to positive solutions. In [12, 13], Lian et al. generalized their existence results to unbounded solutions, and somewhat weakened the conditions in [14]. In 2012, Zhao et al. [15] similarly investigated the solutions to multipoint boundary value problems in Banach spaces on an infinite interval.

Inspired by the works listed above, in this paper, we aim to discuss the $n$ th-order differential equation on a half-line with Sturm-Liouville boundary conditions. To the best of our knowledge, this is the first attempt to find the unbounded solutions to higher-order infinite interval problems by using the upper and lower solution technique. Since, the halfline is noncompact, the discussion is rather involved. We begin with the assumption that there exist a pair of upper and lower solutions for problem (1)-(2), and the nonlinear function $f$ satisfies a Nagumo-type condition. Then, by using the truncation technique and the upper and lower solutions, we estimate a-priori bounds of modified problems. Next, the Schäuder fixed point theorem is used which guarantees the existence of solutions to 
(1)-(2). We also assume two pairs of upper and lower solutions and show that this infinite interval problem has at least three solutions. In the last section, an example is included which illustrates the main result.

\section{Preliminaries}

In this section, we present some definitions and lemmas to be used in the main theorem of this paper.

Consider the space $X$ defined by

$$
X=\left\{u \in C^{n-1}[0,+\infty), \lim _{t \rightarrow+\infty} \frac{u^{(i)}(t)}{v_{i}(t)} \text { exist, } i=0,1, \ldots, n-1\right\}
$$

with the norm $\|\cdot\|$ given by

$$
\|u\|=\max \left\{\|u\|_{0},\|u\|_{1}, \ldots,\|u\|_{n-1}\right\}
$$

where $v_{i}(t)=1+t^{n-1-i}$ and

$$
\|u\|_{i}=\sup _{t \in[0,+\infty)}\left|\frac{u^{(i)}(t)}{v_{i}(t)}\right|, \quad i=0,1, \ldots, n-1
$$

Then $(X,\|\cdot\|)$ is a Banach space.

To obtain a solution of the BVP (1)-(2), we need a mapping whose kernel $G(t, s)$ is the Green function of $-u^{(n)}(t)=0$ with the homogeneous boundary conditions (2), which is given in the following lemma.

Lemma 2.1 Let $e \in L^{1}[0,+\infty)$. Then the linear boundary value problem

$$
\left\{\begin{array}{l}
-u^{(n)}(t)=e(t), \quad 0<t<+\infty \\
u^{(i)}(0)=A_{i}, \quad i=0,1, \ldots, n-3 \\
u^{(n-2)}(0)-a u^{(n-1)}(0)=B \\
u^{(n-1)}(+\infty)=C
\end{array}\right.
$$

has a unique solution given by

$$
u(t)=l(t)+\int_{0}^{+\infty} G(t, s) e(s) d s
$$

where

$$
l(t)=\sum_{k=0}^{n-3} \frac{A_{k}}{k !} t^{k}+\frac{a C+B}{(n-2) !} t^{n-2}+\frac{C}{(n-1) !} t^{n-1},
$$

and

$$
G(t, s)= \begin{cases}\frac{a}{(n-2) !} t^{n-2}+\sum_{k=0}^{n-2} \frac{(-1)^{k}}{(k+1) !(n-2-k) !} s^{k+1} t^{n-2-k}, & 0 \leq s \leq t<+\infty \\ \frac{a}{(n-2) !} t^{n-2}+\frac{1}{(n-1) !} t^{n-1}, & 0 \leq t \leq s<+\infty\end{cases}
$$


Proof Let $v(t)=u^{(n-2)}(t)$. Then from (6), we obtain the Sturm-Liouville boundary value problem

$$
\left\{\begin{array}{l}
-v^{\prime \prime}(t)=e(t), \quad 0<t<+\infty \\
v(0)-a v^{\prime}(0)=B \\
v^{\prime}(+\infty)=C
\end{array}\right.
$$

and the initial value problem

$$
\left\{\begin{array}{l}
u^{(n-2)}(t)=v(t), \quad 0<t<+\infty \\
u^{(i)}(0)=A_{i}, \quad i=0,1, \ldots, n-3 .
\end{array}\right.
$$

Clearly, (9) has a unique solution

$$
v(t)=a C+B+C t+\int_{0}^{+\infty} g(t, s) e(s) d s
$$

where

$$
g(t, s)= \begin{cases}a+s, & 0 \leq s \leq t<+\infty \\ a+t, & 0 \leq t \leq s<+\infty\end{cases}
$$

Now integrating (10) and applying the initial conditions, we obtain (7).

Lemma 2.2 The function $G(t, s)$ defined in $(8)$ is $(n-1)$ times continuously differentiable on $[0,+\infty) \times[0,+\infty)$. For any $s \in[0,+\infty)$, its ith derivative is uniformly continuous in $t$ on any compact interval of $[0,+\infty)$ and is uniformly bounded on $[0,+\infty)$.

Proof We denote $g_{i}(t, s)=\frac{\partial^{i} G(t, s)}{\partial t^{i}}$, which is also used in the later part of the paper. By direct calculations, we have

$$
g_{i}(t, s)= \begin{cases}\frac{a t^{n-2-i}}{(n-2-i) !}+\sum_{k=0}^{n-2-i} \frac{(-1)^{k} s^{k+1} t^{n-2-k-i}}{(k+1) !(n-2-k-i) !}, & 0 \leq s \leq t<+\infty, \\ \frac{a}{(n-2-i) !} t^{n-2-i}+\frac{1}{(n-1-i) !} t^{n-1-i}, & 0 \leq t \leq s<+\infty .\end{cases}
$$

Obviously, $g_{i}(t, s)$ is uniformly continuous in $t$ on any compact interval of $[0,+\infty)$ for any $s \in[0,+\infty)$. Now since, for all integers $k$ and $l$,

$$
\sup _{t \in[0,+\infty)} \frac{t^{k}}{1+t^{l}}= \begin{cases}\frac{l-k}{l}\left(\frac{k}{l-k}\right)^{\frac{k}{l}}, & k<l, \\ 1, & k=l, \\ +\infty, & k>l\end{cases}
$$

from (11), when $s \leq t$, it follows that

$$
\begin{aligned}
\sup _{t \in[0,+\infty)}\left|\frac{g_{i}(t, s)}{v_{i}(t)}\right| & =\sup _{t \in[0,+\infty)}\left|\frac{a t^{n-2-i}}{(n-2-i) ! v_{i}(t)}+\sum_{k=0}^{n-2-i} \frac{(-1)^{k} s^{k+1} t^{n-2-k-i}}{(k+1) !(n-2-k-i) ! v_{i}(t)}\right| \\
& \leq \sup _{t \in[0,+\infty)}\left(\frac{a t^{n-2-i}}{(n-2-i) ! v_{i}(t)}+\sum_{k=0}^{n-2-i} \frac{t^{k+1} t^{n-2-k-i}}{(k+1) !(n-2-k-i) ! v_{i}(t)}\right)
\end{aligned}
$$




$$
\begin{aligned}
& \leq \frac{a}{(n-2-i) !} \sup _{t \in[0,+\infty)} \frac{t^{n-2-i}}{v_{i}(t)}+\frac{n-1-i}{(n-2-i) !} \sup _{t \in[0,+\infty)} \frac{t^{n-1-i}}{v_{i}(t)} \\
& =\frac{a}{(n-1-i) !}(n-2-i)^{\frac{n-2-i}{n-1-i}}+\frac{n-1-i}{(n-2-i) !}
\end{aligned}
$$

and, when $s \geq t$, we have

$$
\begin{aligned}
\sup _{t \in[0,+\infty)}\left|\frac{g_{i}(t, s)}{v_{i}(t)}\right| & =\sup _{t \in[0,+\infty)}\left|\frac{a t^{n-2-i}}{(n-2-i) ! v_{i}(t)}+\frac{t^{n-1-i}}{(n-1-i) ! v_{i}(t)}\right| \\
& \leq \frac{a}{(n-2-i) !} \sup _{t \in[0,+\infty)} \frac{t^{n-2-i}}{v_{i}(t)}+\frac{1}{(n-1-i) !} \sup _{t \in[0,+\infty)} \frac{t^{n-1-i}}{v_{i}(t)} \\
& =\frac{a}{(n-1-i) !}(n-2-i)^{\frac{n-2-i}{n-1-i}}+\frac{1}{(n-1-i) !}
\end{aligned}
$$

Thus, we have

$$
\|G(t, s)\|_{i} \leq \frac{a}{(n-1-i) !}(n-2-i)^{\frac{n-2-i}{n-1-i}}+\frac{n-1-i}{(n-2-i) !}:=K_{i},
$$

for $i=1,2, \ldots, n-1$.

When applying the Schäuder fixed point theorem to prove the existence result, it is necessary to show that the operator is completely continuous. While the usual Arezà-Ascoli lemma fails here due to the non-compactness of $[0,+\infty)$, the following generalization (see $[6,13])$ will be used.

Lemma 2.3 $M \subset X$ is relatively compact if the following conditions hold:

1. all the functions from $M$ are uniformly bounded;

2. all the functions from $M$ are equi-continuous on any compact interval of $[0,+\infty)$;

3. all the functions from $M$ are euqi-convergent at infinity, that is, for any given $\epsilon>0$, there exists a $T=T(\epsilon)>0$ such that for any $u \in M$,

$$
\left|\frac{u^{(i)}(t)}{v_{i}(t)}-\frac{u^{(i)}(+\infty)}{v_{i}(+\infty)}\right|<\epsilon, \quad t>T, i=0,1, \ldots, n-1
$$

Finally, we define lower and upper solutions of (1)-(2), and introduce the Nagumo-type condition.

Definition 2.1 A function $\alpha(t) \in C^{n-1}[0,+\infty) \cap C^{n}(0,+\infty)$ satisfying

$$
\left\{\begin{array}{l}
-\alpha^{(n)}(t) \leq q(t) f\left(t, \alpha(t), \ldots, \alpha^{(n-1)}(t)\right), \quad 0<t<+\infty \\
\alpha^{(i)}(0) \leq A_{i}, \quad i=0,1, \ldots, n-3 \\
\alpha^{(n-2)}(0)-a \alpha^{(n-1)}(0) \leq B \\
\alpha^{(n-1)}(+\infty)<C
\end{array}\right.
$$

is called a lower solution of (1)-(2). If the inequalities are strict, it is called a strict lower solution. 
Definition 2.2 A function $\beta(t) \in C^{n-1}[0,+\infty) \cap C^{n}(0,+\infty)$ satisfying

$$
\left\{\begin{array}{l}
-\beta^{(n)}(t) \geq q(t) f\left(t, \beta(t), \ldots, \beta^{(n-1)}(t)\right), \quad 0<t<+\infty \\
\beta^{(i)}(0) \geq A_{i}, \quad i=0,1, \ldots, n-3, \\
\beta^{(n-2)}(0)-a \beta^{(n-1)}(0) \geq B, \\
\beta^{(n-1)}(+\infty)>C
\end{array}\right.
$$

is called an upper solution of (1)-(2). If the inequalities are strict, it is called a strict upper solution.

Definition 2.3 Let $\alpha, \beta$ be the lower and upper solutions of BVP (1)-(2) satisfying

$$
\alpha^{(i)}(t) \leq \beta^{(i)}(t), \quad i=0,1, \ldots, n-2,
$$

on $[0,+\infty)$. We say $f$ satisfies a Nagumo condition with respect to $\alpha$ and $\beta$ if there exist positive functions $\psi$ and $h \in C[0,+\infty)$ such that

$$
\left|f\left(t, u_{0}, u_{1}, \ldots, u_{n-1}\right)\right| \leq \psi(t) h\left(\left|u_{n-1}\right|\right)
$$

for all $\left(t, u_{0}, u_{1}, \ldots, u_{n-1}\right) \in[0,+\infty) \times[\alpha(t), \beta(t)] \times \cdots \times\left[\alpha^{(n-2)}(t), \beta^{(n-2)}(t)\right] \times \mathbb{R}$ and

$$
\int_{0}^{+\infty} q(s) \psi(s) d s<+\infty, \quad \int^{+\infty} \frac{s}{h(s)} d s=+\infty .
$$

\section{The existence results}

Our existence theory is based on using the unbounded lower and upper solution technique. Here we list some assumptions for convenience.

$\mathrm{H}_{1}$ : BVP (1)-(2) has a pair of upper and lower solutions $\beta, \alpha$ in $X$ with

$$
\alpha^{(i)}(t) \leq \beta^{(i)}(t), \quad i=0,1, \ldots, n-2, t \in[0,+\infty)
$$

and $f \in C\left([0,+\infty) \times \mathbb{R}^{n}, \mathbb{R}\right)$ satisfies the Nagumo condition with respect to $\alpha$ and $\beta$.

$\mathrm{H}_{2}$ : For any fixed $t \in[0,+\infty), u_{n-2}, u_{n-1} \in \mathbb{R}$, when $\alpha^{(i)}(t) \leq u_{i} \leq \beta^{(i)}(t), i=0,1, \ldots, n-3$, the following inequality holds:

$$
\begin{aligned}
f\left(t, \alpha(t), \ldots, \alpha^{(i)}(t), \ldots, u_{n-2}, u_{n-1}\right) & \leq f\left(t, u_{0}, \ldots, u_{i}, \ldots, u_{n-2}, u_{n-1}\right) \\
& \leq f\left(t, \beta(t), \ldots, \beta^{(i)}(t), \ldots, u_{n-2}, u_{n-1}\right) .
\end{aligned}
$$

$\mathrm{H}_{3}$ : There exists a constant $\gamma>1$ such that

$$
\sup _{0 \leq t<+\infty}(1+t)^{\gamma} q(t) \psi(t)<+\infty
$$

where $\psi$ is the function in Nagumo's condition of $f$. 
Lemma 3.1 Suppose conditions $\left(\mathrm{H}_{1}\right)$ and $\left(\mathrm{H}_{3}\right)$ hold. Then there exists a constant $R>0$ such that every solution $u$ of (1)-(2) with

$$
\alpha^{(i)}(t) \leq u \leq \beta^{(i)}(t), \quad i=0,1, \ldots, n-2,0 \leq t<+\infty,
$$

satisfies $\|u\|_{n-1} \leq R$.

Proof Set

$$
\begin{aligned}
& M_{0}=\sup _{0 \leq t<+\infty}(1+t)^{\gamma} q(t) \psi(t), \\
& M_{1}=\sup _{0 \leq t<+\infty} \frac{\beta^{(n-2)}(t)}{(1+t)^{\gamma}}-\inf _{0 \leq t<+\infty} \frac{\alpha^{(n-2)}(t)}{(1+t)^{\gamma}}, \\
& M_{2}=\max \left\{\|\beta\|_{n-2},\|\alpha\|_{n-2}\right\}, \\
& M_{3}=\max \left\{\sup _{\delta \leq t<+\infty} \frac{\beta^{(n-2)}(t)-\alpha^{(n-2)}(0)}{t}, \sup _{\delta \leq t<+\infty} \frac{\beta^{(n-2)}(0)-\alpha^{(n-2)}(t)}{t}\right\},
\end{aligned}
$$

where $\delta>0$ is any arbitrary constant. Choose $R>C$ where $C$ is the nonhomogeneous boundary data, and $\eta \geq M_{3}$ satisfies

$$
\int_{\eta}^{R} \frac{s}{h(s)} d s \geq M_{0}\left(M_{1}+\frac{\gamma}{\gamma-1} M_{2}\right)
$$

If $\left|u^{(n-1)}(t)\right| \leq R$ holds for any $t \in[0,+\infty)$, then the result follows immediately. If not, we claim that $\left|u^{(n-1)}(t)\right|>\eta$ does not hold for all $t \in[0,+\infty)$. Otherwise, without loss of generality, we suppose

$$
u^{(n-1)}(t)>\eta, \quad t \in[0,+\infty)
$$

But then, for any $t \geq \delta>0$, it follows that

$$
\begin{aligned}
\frac{\beta^{(n-2)}(t)-\alpha^{(n-2)}(0)}{t} & \geq \frac{u^{(n-2)}(t)-u^{(n-2)}(0)}{t} \\
& =\frac{1}{t} \int_{0}^{t} u^{(n-1)}(s) d s \\
& >\eta \geq \frac{\beta^{(n-2)}(t)-\alpha^{(n-2)}(0)}{t},
\end{aligned}
$$

which is a contraction. So there must exist $t^{*} \in[0,+\infty)$ such that $\left|u^{(n-1)}\left(t^{*}\right)\right| \leq \eta$. Furthermore, if

$$
\left|u^{(n-1)}(t)\right| \leq \eta, \quad \forall t \in[0,+\infty)
$$

just take $R=\eta$ and then the proof is completed. Finally, there exist $\left[t_{1}, t_{2}\right] \subset[0,+\infty)$ such that $\left|u^{(n-1)}\left(t_{1}\right)\right|=\eta,\left|u^{(n-1)}(t)\right|>\eta, t \in\left(t_{1}, t_{2}\right]$ or $\left|u^{(n-1)}\left(t_{2}\right)\right|=\eta,\left|u^{(n-1)}(t)\right|>\eta, t \in\left[t_{1}, t_{2}\right)$. Sup- 
pose that $u^{(n-1)}\left(t_{1}\right)=\eta, u^{(n-1)}(t)>\eta, t \in\left(t_{1}, t_{2}\right]$. Obviously,

$$
\begin{aligned}
\int_{u^{(n-1)}\left(t_{1}\right)}^{u^{(n-1)}\left(t_{2}\right)} \frac{s}{h(s)} d s & =\int_{t_{1}}^{t_{2}} \frac{u^{(n-1)}(s)}{h\left(u^{(n-1)}(s)\right)} u^{(n)}(s) d s \\
& =\int_{t_{1}}^{t_{2}} \frac{-q(s) f\left(s, u(s), \ldots, u^{(n-1)}(s)\right)}{h\left(u^{(n-1)}(s)\right)} u^{(n-1)}(s) d s \\
& \leq \int_{t_{1}}^{t_{2}} q(s) \psi(s) u^{(n-1)}(s) d s \leq M_{0} \int_{t_{1}}^{t_{2}} \frac{u^{(n-1)}(s)}{(1+s)^{\gamma}} d s \\
& =M_{0}\left(\int_{t_{1}}^{t_{2}}\left(\frac{u^{(n-2)}(s)}{(1+s)^{\gamma}}\right)^{\prime} d s-\int_{t_{1}}^{t_{2}} u^{(n-2)}(s)\left(\frac{1}{(1+s)^{\gamma}}\right)^{\prime} d s\right) \\
& \leq M_{0}\left(M_{1}+M_{2} \int_{0}^{+\infty} \frac{\gamma}{(1+s)^{\gamma}} d s\right) \leq \int_{\eta}^{R} \frac{s}{h(s)} d s,
\end{aligned}
$$

from which one concludes that $u^{(n-1)}\left(t_{2}\right) \leq R$. Since $t_{1}$ and $t_{2}$ are arbitrary, we have $u^{(n-1)}(t) \leq R$ if $u^{(n-1)}(t) \geq \eta$ for $t \in[0,+\infty)$. In a similarly way, we can show that $u^{(n-1)}(t) \geq$ $-R$, if $u^{(n-1)}(t) \leq-\eta$ for $t \in[0,+\infty)$.

Therefore there exists a $R>0$, just related with $\alpha, \beta$, and $\psi, h$ under the Nagumo condition of $f$, such that $\|u\|_{n-1} \leq R$.

Remark 3.1 Similarly, we can prove that

$$
\left|\beta^{(n-1)}(t)\right| \leq R, \quad\left|\alpha^{(n-1)}(t)\right| \leq R, \quad t \in[0,+\infty)
$$

Remark 3.2 The Nagumo condition plays a key role in estimating the prior bound for the $(n-1)$ th derivative of the solution of BVP (1)-(2). Since the upper and lower solutions are in $X, \alpha^{(n-2)}(t)$ and $\beta^{(n-2)}(t)$ may be asymptotic linearly at infinity.

Theorem 3.2 Suppose the conditions $\left(\mathrm{H}_{1}\right)-\left(\mathrm{H}_{3}\right)$ hold. Then BVP (1)-(2) has at least one solution $u \in C^{n-1}[0,+\infty) \cap C^{n}(0,+\infty)$ satisfying

$$
\alpha^{(i)}(t) \leq u^{(i)}(t) \leq \beta^{(i)}(t), \quad i=0,1, \ldots, n-2, t \in[0,+\infty)
$$

Moreover, there exists a $R>0$ such that $\|u\|_{n-1} \leq R$.

Proof Let $R>0$ be the same as in Lemma 3.1. Define the auxiliary functions $f_{0}, f_{1}, \ldots, f_{n-2}$, and $F:[0,+\infty) \times \mathbb{R}^{n} \rightarrow \mathbb{R}$ as

$$
\begin{aligned}
& f_{0}\left(t, u_{0}, u_{1}, \ldots, u_{n-1}\right)= \begin{cases}f\left(t, \beta, u_{1}, \ldots, u_{n-1}\right), & u_{0}>\beta(t), t \in[0,+\infty), \\
f\left(t, u_{0}, u_{1}, \ldots, u_{n-1}\right), & \alpha(t) \leq u_{0} \leq \beta(t), t \in[0,+\infty), \\
f\left(t, \alpha, u_{1}, \ldots, u_{n-1}\right), & u_{0}<\alpha(t), t \in[0,+\infty),\end{cases} \\
& f_{i}\left(t, u_{0}, \ldots, u_{i}, \ldots, u_{n-1}\right) \\
& \quad= \begin{cases}f_{i-1}\left(t, u_{0}, \ldots, \beta^{(i)}, \ldots, u_{n-1}\right), & u_{i}>\beta^{(i)}(t), t \in[0,+\infty), \\
f_{i-1}\left(t, u_{0}, \ldots, u_{i}, \ldots, u_{n-1}\right), & \alpha^{(i)}(t) \leq u_{i} \leq \beta^{(i)}(t), t \in[0,+\infty), \\
f_{i-1}\left(t, u_{0}, \ldots, \alpha^{(i)}, \ldots, u_{n-1}\right), & u_{i}<\alpha^{(i)}(t), t \in[0,+\infty),\end{cases}
\end{aligned}
$$


for $i=1,2, \ldots, n-3$, and

$$
\begin{gathered}
f_{n-2}\left(t, u_{0}, \ldots, u_{n-2}, u_{n-1}\right)=\left\{\begin{array}{c}
f_{n-3}\left(t, u_{0}, \ldots, \beta^{(n-2)}, u_{n-1}\right)-\frac{u_{n-2}-\beta^{(n-2)}(t)}{1+\left|u_{n-2}-\beta^{(n-2)}(t)\right|}, \\
u_{n-2}>\beta^{(n-2)}(t), t \in[0,+\infty), \\
f_{n-3}\left(t, u_{0}, \ldots, u_{n-2}, u_{n-1}\right), \\
\alpha^{(n-2)}(t) \leq u_{n-2} \leq \beta^{(n-2)}(t), t \in[0,+\infty), \\
f_{n-3}\left(t, u_{0}, \ldots, \alpha^{(n-2)}(t), u_{n-1}\right)+\frac{u_{n-2}-\alpha^{(n-2)}(t)}{1+\left|y-\alpha^{(n-2)}(t)\right|}, \\
u_{n-2}<\alpha^{(n-2)}(t), t \in[0,+\infty),
\end{array}\right. \\
F\left(t, u_{0}, \ldots, u_{n-2}, u_{n-1}\right)=\left\{\begin{array}{cc}
f_{n-2}\left(t, u_{0}, \ldots, u_{n-2}, R\right), & u_{n-1}>R, t \in[0,+\infty), \\
f_{n-2}\left(t, u_{0}, \ldots, u_{n-2}, u_{n-1}\right), & \left|u_{n-1}\right| \leq R, t \in[0,+\infty), \\
f_{n-2}\left(t, u_{0}, \ldots, u_{n-2},-R\right), & u_{n-1}<-R, t \in[0,+\infty) .
\end{array}\right.
\end{gathered}
$$

Consider the modified differential equation with the truncated function

$$
-u^{(n)}(t)=q(t) F\left(t, u(t), \ldots, u^{(n-1)}(t)\right), \quad t \in(0,+\infty)
$$

with the boundary conditions (2). To complete the proof, it suffices to show that problem (16)-(2) has at least one solution $u$ satisfying

$$
\alpha^{(i)}(t) \leq u^{(i)}(t) \leq \beta^{(i)}(t), \quad i=0,1, \ldots, n-2, t \in[0,+\infty)
$$

and

$$
\left|u^{(n-1)}(t)\right| \leq R, \quad t \in[0,+\infty) .
$$

We divide the proof into the following two steps.

Step 1: By contradiction we shall show that every solution $u$ of problem (16)-(2) satisfy (17) and (18).

Suppose the right hand inequality in (17) does not hold for $i=n-2$. Set $\omega(t)=u^{(n-2)}(t)-$ $\beta^{(n-2)}(t)$, then

$$
\sup _{0 \leq t<+\infty} \omega(t)>0
$$

Case I. $\lim _{t \rightarrow 0^{+}} \omega(t)=\sup _{0 \leq t<+\infty} \omega(t)>0$.

Obviously, we have $\omega^{\prime}\left(0^{+}\right) \leq 0$. By the boundary conditions, it follows that

$$
\omega^{\prime}\left(0^{+}\right)=u^{(n-1)}(0)-\beta^{(n-1)}(0) \geq \frac{1}{a}\left(u^{(n-2)}(0)-\beta^{(n-2)}(0)\right)=\frac{1}{a} \omega(0)>0,
$$

which is a contradiction.

Case II. There exists a $t^{*} \in(0,+\infty)$ such that $\omega\left(t^{*}\right)=\sup _{0 \leq t<+\infty} \omega(t)>0$.

Clearly, we have

$$
\omega\left(t^{*}\right)>0, \quad \omega^{\prime}\left(t^{*}\right)=0, \quad \omega^{\prime \prime}\left(t^{*}\right) \leq 0 .
$$


On the other hand,

$$
\begin{aligned}
u^{(n)}\left(t^{*}\right)= & -q\left(t^{*}\right) F\left(t^{*}, u\left(t^{*}\right), \ldots, u^{(n-2)}\left(t^{*}\right), u^{(n-1)}\left(t^{*}\right)\right) \\
= & -q\left(t^{*}\right) f_{n-2}\left(t^{*}, u\left(t^{*}\right), \ldots, u^{(n-2)}\left(t^{*}\right), \beta^{(n-1)}\left(t^{*}\right)\right) \\
= & -q\left(t^{*}\right)\left(f_{n-3}\left(t^{*}, u\left(t^{*}\right), \ldots, \beta^{(n-2)}\left(t^{*}\right), \beta^{(n-1)}\left(t^{*}\right)\right)\right. \\
& \left.-\frac{u^{n-2}\left(t^{*}\right)-\beta^{(n-2)}\left(t^{*}\right)}{1+\left|u^{n-2}\left(t^{*}\right)-\beta^{(n-2)}\left(t^{*}\right)\right|}\right) .
\end{aligned}
$$

Subcase i. If $u^{(n-3)}\left(t^{*}\right)>\beta^{(n-3)}\left(t^{*}\right)$, from the definition of $f_{n-3}$, we obtain

$$
\begin{aligned}
u^{(n)}\left(t^{*}\right)= & -q\left(t^{*}\right) f_{n-4}\left(t^{*}, u\left(t^{*}\right), \ldots, \beta^{(n-3)}\left(t^{*}\right), \beta^{(n-2)}\left(t^{*}\right), \beta^{(n-1)}\left(t^{*}\right)\right) \\
& +q\left(t^{*}\right) \frac{u^{n-2}\left(t^{*}\right)-\beta^{(n-2)}\left(t^{*}\right)}{1+\left|u^{n-2}\left(t^{*}\right)-\beta^{(n-2)}\left(t^{*}\right)\right|} .
\end{aligned}
$$

Subcase ii. If $u^{(n-3)}\left(t^{*}\right) \leq \beta^{(n-3)}\left(t^{*}\right)$, from the conditions $\left(\mathrm{H}_{2}\right)$, we find

$$
\begin{aligned}
u^{(n)}\left(t^{*}\right) \geq & -q\left(t^{*}\right) f_{n-4}\left(t^{*}, u\left(t^{*}\right), \ldots, \beta^{(n-3)}\left(t^{*}\right), \beta^{(n-2)}\left(t^{*}\right), \beta^{(n-1)}\left(t^{*}\right)\right) \\
& +q\left(t^{*}\right) \frac{u^{n-2}\left(t^{*}\right)-\beta^{(n-2)}\left(t^{*}\right)}{1+\left|u^{n-2}\left(t^{*}\right)-\beta^{(n-2)}\left(t^{*}\right)\right|} .
\end{aligned}
$$

Similarly following the above argument, we could discuss the other two cases $u^{(i)}\left(t^{*}\right)>$ $\beta^{(i)}\left(t^{*}\right)$ or $u^{(i)}\left(t^{*}\right) \leq \beta^{(i)}\left(t^{*}\right), i=n-4, n-5, \ldots, 1,0$, and we have the following inequality:

$$
u^{(n)}\left(t^{*}\right) \geq-q\left(t^{*}\right)\left(f\left(t^{*}, \beta\left(t^{*}\right), \ldots, \beta^{(n-2)}\left(t^{*}\right), \beta^{(n-1)}\left(t^{*}\right)\right)-\frac{u^{n-2}\left(t^{*}\right)-\beta^{(n-2)}\left(t^{*}\right)}{1+\left|u^{n-2}\left(t^{*}\right)-\beta^{(n-2)}\left(t^{*}\right)\right|}\right) .
$$

Thus,

$$
\omega^{\prime \prime}\left(t^{*}\right) \geq q\left(t^{*}\right) \frac{u^{n-2}\left(t^{*}\right)-\beta^{(n-2)}\left(t^{*}\right)}{1+\left|u^{n-2}\left(t^{*}\right)-\beta^{(n-2)}\left(t^{*}\right)\right|}>0,
$$

which contradicts (19).

Thus, $u^{(n-2)}(t) \leq \beta^{(n-2)}(t), t \in[0,+\infty)$. Similarly, we can show that $u^{(n-2)}(t) \geq \alpha^{(n-2)}(t)$, $t \in[0,+\infty)$. Integrating this inequality and using the boundary conditions in (2), (13), and (14), we obtain the inequality (17). Inequality (18) then follows from Lemma 3.1. In conclusion, $u$ is the required solution to BVP (1)-(2).

Step 2. Problem (16)-(2) has a solution $u$.

Consider the operator $T: X \rightarrow X$ defined by

$$
T u(t)=l(t)+\int_{0}^{+\infty} G(t, s) q(s) F\left(s, u(s), \ldots, u^{(n-1)}(s)\right) d s .
$$

Lemma 2.1 shows that the fixed points of $T$ are the solutions of BVP (16)-(2). Next we shall prove that $T$ has at least one fixed point by using the Schäuder fixed point theorem. For this, it is enough to show that $T: X \rightarrow X$ is completely continuous.

(1) $T: X \rightarrow X$ is well defined. 
For any $u \in X$, by direct calculation, we find

$$
\begin{aligned}
(T u)^{(i)}(t)= & \sum_{k=i}^{n-3} \frac{A_{k} t^{k-i}}{(k-i) !}+\frac{(a C+B) t^{n-2-i}}{(n-2-i) !}+\frac{C t^{n-1-i}}{(n-1-i) !} \\
& +\int_{0}^{+\infty} g_{i}(t, s) q(s) F\left(s, u(s), \ldots, u^{(n-1)}(s)\right) d s, \quad i=0,1, \ldots, n-1 .
\end{aligned}
$$

Obviously, Tu $\in C^{n-1}[0,+\infty)$. Further, because

$$
\left|\int_{0}^{+\infty} q(s) F\left(s, u(s), \ldots, u^{(n-1)}(s)\right) d s\right| \leq \int_{0}^{+\infty} q(s)(H \psi(s)+1) d s<+\infty
$$

where $H=\max _{0 \leq s \leq\|u\|} h(s)$, the Lebesgue dominated convergent theorem implies that

$$
\begin{aligned}
\lim _{t \rightarrow+\infty} \frac{(T u)^{(i)}(t)}{v_{i}(t)}= & \lim _{t \rightarrow+\infty}\left(\sum_{k=i}^{n-3} \frac{A_{k} t^{k-i}}{(k-i) ! v_{i}(t)}+\frac{(a C+B) t^{n-2-i}}{(n-2-i) ! v_{i}(t)}+\frac{C t^{n-1-i}}{(n-1-i) ! v_{i}(t)}\right) \\
& +\int_{0}^{+\infty} \lim _{t \rightarrow+\infty} \frac{g_{i}(t, s)}{v_{i}(t)} q(s) F\left(s, u(s), \ldots, u^{(n-1)}(s)\right) d s \\
= & \frac{C}{(n-1-i) !}+\int_{0}^{+\infty} \frac{1}{(n-1-i) !} q(s) F\left(s, u(s), \ldots, u^{(n-1)}(s)\right) d s \\
& <+\infty .
\end{aligned}
$$

Thus, $T u \in X$.

(2) $T: X \rightarrow X$ is continuous.

For any convergent sequence $u_{m} \rightarrow u$ in $X$, there exists $r_{1}>0$ such that $\sup _{m \in N}\left\|u_{m}\right\| \leq$ $r_{1}$. Thus, as in (21), we have

$$
\begin{aligned}
\left\|T u_{m}-T u\right\|_{i}= & \sup _{t \in[0,+\infty)}\left|\frac{\left(T u_{m}\right)^{(i)}(t)}{v_{i}(t)}-\frac{(T u)^{(i)}(t)}{v_{i}(t)}\right| \\
\leq & \int_{0}^{+\infty} \sup _{0 \leq t<+\infty} \frac{g_{i}(t, s)}{v_{i}(t)} \\
& \cdot q(s)\left|F\left(s, u_{m}(s), \ldots, u_{m}^{(n-1)}(s)\right)-F\left(s, u(s), \ldots, u^{(n-1)}(s)\right)\right| d s \\
\leq & \int_{0}^{+\infty} K_{i} q(s)\left|F\left(s, u_{m}(s), \ldots, u_{m}^{(n-1)}(s)\right)-F\left(s, u(s), \ldots, u^{(n-1)}(s)\right)\right| d s \\
\rightarrow & 0, \quad \text { as } m \rightarrow+\infty, i=0,1, \ldots, n-1,
\end{aligned}
$$

and hence $T: X \rightarrow X$ is continuous.

(3) $T: X \rightarrow X$ is compact.

For this it suffices to show that $T$ maps bounded subsets of $X$ into relatively compact sets. Let $B$ be any bounded subset of $X$, then there exists $r_{2}>0$ such that $\|u\| \leq r_{2}, \forall u \in B$. For any $u \in B, i=0,1, \ldots, n-1$, we have

$$
\begin{aligned}
\sup _{t \in[0,+\infty)}\left|\frac{(T u)^{(i)}(t)}{v_{i}(t)}\right| \leq & \sum_{k=i}^{n-3} \frac{\left|A_{k}\right|}{(k-i) !} \sup _{t \in[0,+\infty)}\left|\frac{t^{k-i}}{v_{i}(t)}\right|+\frac{(a C+B)}{(n-2-i) !} \sup _{t \in[0,+\infty)}\left|\frac{t^{n-2-i}}{v_{i}(t)}\right| \\
& +\frac{C}{(n-1-i) !} \sup _{t \in[0,+\infty)}\left|\frac{t^{n-1-i}}{v_{i}(t)}\right|
\end{aligned}
$$




$$
\begin{gathered}
\quad+\int_{0}^{+\infty} \sup _{t \in[0,+\infty)}\left|\frac{g_{i}(t, s)}{v_{i}(t)}\right| q(s) F\left(s, u(s), \ldots, u^{(n-1)(s)}\right) d s \\
\leq L_{i}+\int_{0}^{+\infty} K_{i} q(s)\left(r_{3} \psi(s)+1\right) d s=r_{4, i}<+\infty,
\end{gathered}
$$

where

$$
\begin{aligned}
L_{i}= & \sum_{k=i}^{n-3} \frac{\left|A_{k}\right|(n-1-k)}{(k-i) !(n-1-i)} \cdot\left(\frac{k-i}{n-1-k}\right)^{\frac{k-i}{n-1-i}} \\
& +\frac{(a C+B)}{(n-3-i) !} \cdot\left(\frac{1}{n-2-i}\right)^{\frac{1}{n-1-i}}+\frac{|C|}{(n-1-i) !},
\end{aligned}
$$

where $r_{3}=\max _{0 \leq s \leq r_{2}} h(s)$, and thus $T B$ is uniformly bounded. Further, for any $T>0$, if $t_{1}, t_{2} \in[0, T]$, we have

$$
\begin{aligned}
& \left|\frac{(T u)^{(i)}\left(t_{1}\right)}{v_{i}\left(t_{1}\right)}-\frac{(T u)^{(i)}\left(t_{2}\right)}{v_{i}\left(t_{2}\right)}\right| \\
& \leq\left|\frac{1}{v_{i}\left(t_{1}\right)}\left((T u)^{(i)}\left(t_{1}\right)-(T u)^{(i)}\left(t_{2}\right)\right)\right|+\left|(T u)^{(i)}\left(t_{2}\right)\left(\frac{1}{v_{i}\left(t_{1}\right)}-\frac{1}{v_{i}\left(t_{2}\right)}\right)\right| \\
& \leq \sum_{k=i}^{n-3} \frac{\left|A_{k}\right|}{(k-i) !}\left|t_{1}^{k-i}-t_{2}^{k-i}\right|+\frac{|a C+B|}{(n-2-i) !}\left|t_{1}^{n-2-i}-t_{2}^{n-2-i}\right|+|C|\left|t_{1}^{n-2-i}-t_{2}^{n-2-i}\right| \\
& \quad+\int_{0}^{+\infty}\left|g_{i}\left(t_{1}, s\right)-g_{i}\left(t_{2}, s\right)\right| q(s)\left|F\left(s, u(s), \ldots, u^{(n-1)}(s)\right)\right| d s+r_{4, i}\left|t_{2}^{n-1-i}-t_{1}\right|
\end{aligned}
$$

$\rightarrow 0$, uniformly as $t_{1} \rightarrow t_{2}$,

that is, $T B$ is equi-continuous. From Lemma 2.3 , it follows that if $T B$ is equi-convergent at infinity, then $T B$ is relatively compact. In fact, we have

$$
\begin{aligned}
& \left|\frac{(T u)^{i}(t)}{v_{i}(t)}-\lim _{t \rightarrow+\infty} \frac{(T u)^{i}(t)}{v_{i}(t)}\right| \\
& \quad \leq\left|\frac{l^{(i)}(t)}{v_{i}(t)}-\frac{C}{(n-1-i) !}\right|+\int_{0}^{+\infty}\left|\frac{g_{i}(t, s)}{v_{i}(t)}-\frac{1}{(n-1-i) !}\right| q(s)\left(r_{3} \psi(s)+1\right) d s \\
& \quad \rightarrow 0, \quad \text { uniformly as } t \rightarrow+\infty,
\end{aligned}
$$

and, therefore, $T: X \rightarrow X$ is completely continuous. The Schäuder fixed point theorem now ensures that the operator $T$ has a fixed point, which is a solution of the BVP (1)-(2).

Remark 3.3 The upper and lower solutions are more strict at infinity than usually assumed. For such right boundary conditions, it is not easy to estimate the sign of $\omega^{\prime \prime}(+\infty)$ even though we have $\omega(+\infty)>0, \omega^{\prime}(+\infty)=0$, where $\omega(t)=u^{n-2}(t)-\beta^{n-2}(t)$ or $\omega(t)=$ $u^{n-2}(t)-\alpha^{n-2}(t)$. It remains unsettled whether this strict inequality can be weakened.

\section{The multiplicity results}

In this section assuming two pairs of upper and lower solutions, we shall prove the existence of at least three solutions for our infinite interval problem. 
Theorem 4.1 Suppose that the following condition holds.

$\mathrm{H}_{4}$ : BVP (1)-(2) has two pairs of upper and lower solution $\beta_{j}, \alpha_{j}, j=1,2$ in $X$ with $\alpha_{2}, \beta_{1}$ strict, and

$$
\alpha_{1}^{(i)}(t) \leq \alpha_{2}^{(i)}(t) \leq \beta_{2}^{(i)}(t), \quad \alpha_{1}^{(i)}(t) \leq \beta_{1}^{(i)}(t) \leq \beta_{2}^{(i)}(t), \quad \alpha_{2}^{(i)}(t) \not \leq \beta_{1}^{(i)}(t),
$$

for $i=0,1, \ldots, n-2, t \in[0,+\infty)$, and $f \in C\left([0,+\infty) \times \mathbb{R}^{n}, \mathbb{R}\right)$ satisfies the Nagumo condition with respect to $\alpha_{1}$ and $\beta_{2}$.

Suppose further that conditions $\left(\mathrm{H}_{2}\right)$ and $\left(\mathrm{H}_{3}\right)$ hold with $\alpha$ and $\beta$ replaced by $\alpha_{1}$ and $\beta_{2}$, respectively. Then the problem (1)-(2) has at least three solutions $u_{1}, u_{2}$, and $u_{3}$ satisfying

$$
\alpha_{j}^{(i)}(t) \leq u_{j}^{(i)}(t) \leq \beta_{j}^{(i)}(t) \quad(j=1,2), \quad u_{3}^{(i)}(t) \not \leq \beta_{1}^{(i)}(t) \quad \text { and } \quad u_{3}^{(i)}(t) \nsupseteq \alpha_{2}^{(i)}(t),
$$

for $i=0,1, \ldots, n-2, t \in[0,+\infty)$.

Proof Define the truncated function $F_{1}$, the same as $F$ in Theorem 3.2 with $\alpha$ replaced by $\alpha_{1}$ and $\beta$ by $\beta_{2}$, respectively. Consider the modified differential equation

$$
-u^{(n)}(t)=q(t) F_{1}\left(t, u(t), \ldots, u^{(n-1)}(t)\right), \quad 0<t<+\infty,
$$

with boundary conditions (2). Similarly to Theorem 3.2, it suffices to show that problem (22)-(2) has at least three solutions. To this end, define the mapping $T_{1}: X \rightarrow X$ as follows:

$$
T_{1} u(t)=l(t)+\int_{0}^{+\infty} G(t, s) q(s) F_{1}\left(s, u(s), \ldots, u^{(n-1)}(s)\right) d s .
$$

Clearly, $T_{1}$ is completely continuous. By using the degree theory, we will show that $T_{1}$ has at least three fixed points which coincide with the solutions of (22)-(2).

Let

$$
N>\left\{\max _{0 \leq i \leq n-1} L_{i}+\max _{0 \leq i \leq n-1} K_{i} \int_{0}^{+\infty} q(s)\left(H_{R} \psi(s)+1\right) d s,\left\|\alpha_{1}\right\|,\left\|\beta_{2}\right\|\right\},
$$

where $L_{i}$ and $K_{i}$ are defined as above, and $H_{r}=\max _{0 \leq s \leq R} h(s)$. Set $\Omega=\{u \in X,\|u\|<N\}$. Then for any $u \in \bar{\Omega}$, it follows that

$$
\begin{aligned}
\left\|T_{1} u\right\|= & \max _{0 \leq i \leq n-1}\left\{\sup _{t \in[0,+\infty)}\left|\frac{(T u)^{(i)}(t)}{v_{i}(t)}\right|\right\} \\
\leq & \max _{0 \leq i \leq n-1}\left\{\sum_{k=i}^{n-3} \frac{\left|A_{k}\right|}{(k-i) !} \sup _{t \in[0,+\infty)}\left|\frac{t^{k-i}}{v_{i}(t)}\right|+\frac{(a C+B)}{(n-2-i) !} \sup _{t \in[0,+\infty)}\left|\frac{t^{n-2-i}}{v_{i}(t)}\right|\right. \\
& +\frac{C}{(n-1-i) !} \sup _{t \in[0,+\infty)}\left|\frac{t^{n-1-i}}{v_{i}(t)}\right| \\
& \left.+\int_{0}^{+\infty} \sup _{t \in[0,+\infty)}\left|\frac{g_{i}(t, s)}{v_{i}(t)}\right| q(s) F_{1}\left(s, u(s), \ldots, u^{(n-1)(s)}\right) d s\right\} \\
\leq & \max _{0 \leq i \leq n-1}\left\{L_{i}+\int_{0}^{+\infty} K_{i} q(s)\left(H_{R} \psi(s)+1\right) d s\right\}<N
\end{aligned}
$$

and hence $T \Omega \subset \Omega$, which implies that $\operatorname{deg}\left(I-T_{1}, \Omega, 0\right)=1$. 
Set

$$
\begin{aligned}
& \Omega_{\alpha_{2}}=\left\{u \in \Omega, u^{(n-2)}(t)>\alpha_{2}^{(n-2)}(t), t \in[0,+\infty)\right\}, \\
& \Omega^{\beta_{1}}=\left\{u \in \Omega, u^{(n-2)}(t)<\beta_{1}^{(n-2)}(t), t \in[0,+\infty)\right\} .
\end{aligned}
$$

Because $\alpha_{2}^{(n-2)} \not \leq \beta_{1}^{(n-2)}, \alpha_{1}^{(n-2)} \leq \alpha_{2}^{(n-2)} \leq \beta_{2}^{(n-2)}$ and $\alpha_{1}^{(n-2)} \leq \beta_{1}^{(n-2)} \leq \beta_{2}^{(n-2)}$, we have

$$
\Omega_{\alpha_{2}} \neq \emptyset, \quad \Omega^{\beta_{1}} \neq \emptyset, \quad \Omega \backslash \overline{\Omega_{\alpha_{2}} \cup \Omega^{\beta_{1}}} \neq \emptyset, \quad \Omega_{\alpha_{2}} \cap \Omega^{\beta_{1}}=\emptyset .
$$

Now since $\alpha_{2}, \beta_{1}$ are strict lower and upper solutions, there is no solution in $\partial \Omega_{\alpha_{2}} \cup \partial \Omega^{\beta_{1}}$. Therefore

$$
\begin{aligned}
\operatorname{deg}\left(I-T_{1}, \Omega, 0\right)= & \operatorname{deg}\left(I-T_{1}, \Omega \backslash \overline{\Omega_{\alpha_{2}} \cup \Omega^{\beta_{1}}}, 0\right) \\
& +\operatorname{deg}\left(I-T_{1}, \Omega_{\alpha_{2}}, 0\right)+\operatorname{deg}\left(I-T_{1}, \Omega^{\beta_{1}}, 0\right) .
\end{aligned}
$$

Next we will show that

$$
\operatorname{deg}\left(I-T_{1}, \Omega_{\alpha_{2}}, 0\right)=\operatorname{deg}\left(I-T_{1}, \Omega^{\beta_{1}}, 0\right)=1 .
$$

For this, we define another mapping $T_{2}: \bar{\Omega} \rightarrow \bar{\Omega}$ by

$$
T_{2} u(t)=l(t)+\int_{0}^{+\infty} G(t, s) q(s) F_{2}\left(s, u(s), \ldots, u^{(n-1)}(s)\right) d s
$$

where the function $F_{2}$ is similar to $F_{1}$ except changing $\alpha_{1}$ to $\alpha_{2}$. Similar to the proof of Theorem 3.2, we find that $u$ is a fixed point of $T_{2}$ only if $\alpha_{2}(t) \leq u(t) \leq \beta_{2}(t)$. So $\operatorname{deg}(I-$ $\left.T_{2}, \Omega \backslash \overline{\Omega_{\alpha_{2}}}, 0\right)=0$. From the Schäuder fixed point theorem and $T_{2} \bar{\Omega} \subset \Omega$, we have $\operatorname{deg}(I-$ $\left.T_{2}, \Omega, 0\right)=1$. Furthermore,

$$
\begin{aligned}
\operatorname{deg}\left(I-T_{1}, \Omega_{\alpha_{2}}, 0\right) & =\operatorname{deg}\left(I-T_{2}, \Omega_{\alpha_{2}}, 0\right) \\
& =\operatorname{deg}\left(I-T_{2}, \Omega, 0\right)+\operatorname{deg}\left(I-T_{2}, \Omega \backslash \overline{\Omega_{\alpha_{2}}}, 0\right)=1 .
\end{aligned}
$$

Similarly, we have $\operatorname{deg}\left(I-T_{1}, \Omega^{\beta_{1}}, 0\right)=1$, and then

$$
\operatorname{deg}\left(I-T_{1}, \Omega \backslash \overline{\Omega_{\alpha_{2}} \cup \Omega^{\beta_{1}}}, 0\right)=-1 .
$$

Finally, using the properties of the degree, we conclude that $T_{1}$ has at least three fixed points $u_{1} \in \Omega_{\alpha_{2}}, u_{2} \in \Omega^{\beta_{1}}$, and $u_{3} \in \Omega \backslash \overline{\Omega_{\alpha_{2}} \cup \Omega^{\beta_{1}}}$.

\section{An example}

Example Consider the second-order differential equation with Sturm-Liouville boundary conditions

$$
\left\{\begin{array}{l}
u^{\prime \prime}(t)-\frac{3\left(u^{\prime}(t)+1\right)\left(u^{\prime}(t)-\frac{1}{3}\right) \sqrt[3]{u^{\prime}(t)}}{(1+t)^{2}}-\frac{u^{\prime}(t)-\frac{1}{2}}{(1+t)^{2}}=0, \quad 0<t<+\infty \\
u(0)-3 u^{\prime}(0)=0, \quad u^{\prime}(+\infty)=\frac{1}{2}
\end{array}\right.
$$


Clearly, BVP (23) is a particular case of problem (1)-(2) with

$$
\begin{aligned}
& q(t)=\frac{1}{(1+t)^{2}}, \\
& f\left(t, u_{0}, u_{1}\right)=-2\left(u_{1}+1\right)\left(u_{1}-\frac{1}{3}\right) \sqrt[3]{u_{1}}-\left(u_{1}-\frac{1}{2}\right), \\
& a=3>0, \quad B=0, \quad \text { and } \quad C=\frac{1}{2} .
\end{aligned}
$$

We let

$$
\alpha_{1}(t)=-t-4, \quad \alpha_{2}(t)=\frac{t}{3}, \quad t \in[0,+\infty) .
$$

Then $\alpha_{1}, \alpha_{2} \in C^{2}[0,+\infty)$, and $\alpha_{1}^{\prime}(t)=-1, \alpha_{1}^{\prime \prime}(t)=0, \alpha_{2}^{\prime}(t)=\frac{1}{3}, \alpha_{2}^{\prime \prime}(t)=0$. Moreover, we have

$$
\left\{\begin{array}{l}
\alpha_{1}^{\prime \prime}(t)+f\left(t, \alpha_{1}(t), \alpha_{1}^{\prime}(t)\right)=-\frac{-1-\frac{1}{2}}{(1+t)^{2}}>0, \quad t \in(0,+\infty), \\
\alpha_{1}(0)-3 \alpha_{1}^{\prime}(0)=-1<0, \quad \alpha_{1}^{\prime}(+\infty)=-1<\frac{1}{2},
\end{array}\right.
$$

and

$$
\left\{\begin{array}{l}
\alpha_{2}^{\prime \prime}(t)+f\left(t, \alpha_{2}(t), \alpha_{2}^{\prime}(t)\right)=-\frac{\frac{1}{3}-\frac{1}{2}}{(1+t)^{2}}>0, \quad t \in(0,+\infty) \\
\alpha_{2}(0)-3 \alpha_{2}^{\prime}(0)=-1<0, \quad \alpha_{2}^{\prime}(+\infty)=\frac{1}{3}<\frac{1}{2}
\end{array}\right.
$$

Thus, $\alpha_{1}$ and $\alpha_{2}$ are strict lower solutions of problem (23).

Now we take

$$
\beta_{1}(t)=\left\{\begin{array}{ll}
-\frac{t}{4}, & 0 \leq t \leq 1, \\
\frac{3}{4} t-1, & t>1,
\end{array} \quad \beta_{2}(t)=t+4, \quad t \in[0,+\infty) .\right.
$$

Then $\beta_{1} \in C^{2}[0,1) \cup C^{2}(1,+\infty), \beta_{2} \in C^{2}[0,+\infty)$,

$$
\left\{\begin{array}{l}
\beta_{1}^{\prime \prime}(t)+f\left(t, \beta_{1}(t), \beta_{1}^{\prime}(t)\right)=\frac{-\frac{21}{16} \cdot \sqrt[3]{\frac{1}{4}}+\frac{3}{4}}{(1+t)^{2}}<0, \quad t \in(0,1), \\
\beta_{1}^{\prime \prime}(t)+f\left(t, \beta_{1}(t), \beta_{1}^{\prime}(t)\right)=\frac{-\frac{35}{16} \cdot \sqrt[3]{\frac{3}{4}}-\frac{1}{4}}{(1+t)^{2}}<0, \quad t \in(1,+\infty), \\
\beta_{1}(0)-3 \beta_{1}^{\prime}(0)=\frac{3}{4}>0, \\
\beta_{1}^{\prime}(+\infty)=\frac{3}{4}>\frac{1}{2}
\end{array}\right.
$$

and

$$
\left\{\begin{array}{l}
\beta_{2}^{\prime \prime}(t)+f\left(t, \beta_{2}(t), \beta_{2}^{\prime}(t)\right)=-\frac{9}{2(1+t)^{2}}<0, \quad t \in(0,+\infty) \\
\beta_{2}(0)-3 \beta_{2}^{\prime}(0)=1>0 \\
\beta_{2}^{\prime}(+\infty)=1>\frac{1}{2}
\end{array}\right.
$$

Thus, $\beta_{1}$ and $\beta_{2}$ are strict upper solutions of problem (23). Further, it follows that

$$
\alpha_{1}(t) \leq \alpha_{2}(t) \leq \beta_{2}(t), \quad \alpha_{1}(t) \leq \beta_{1}(t) \leq \beta_{2}(t), \quad \alpha_{2}(t) \not \leq \beta_{1}(t), \quad t \in[0,+\infty) .
$$


Moreover, for every $\left(t, u_{0}, u_{1}\right) \in[0,+\infty) \times[-t-4, t+4] \times[-1,1]$, we find that $f\left(t, u_{0}, u_{1}\right)$ is bounded. Finally, take $\psi(t)=1, h(s)=\max _{-1 \leq r \leq 1}\left|3(r+1)\left(r-\frac{1}{3}\right) \sqrt[3]{r}+\left(r-\frac{1}{2}\right)\right|$. Hence, all conditions in Theorem 4.1 are satisfied and therefore problem (23) has at least three solutions.

\section{Competing interests}

The authors declare that they have no competing interests.

Authors' contributions

All authors contributed equally to the writing of this paper. All authors read and approved the final manuscript.

\section{Author details}

${ }^{1}$ School of Science, China University of Geosciences, Beijing, 100083, PR China. ${ }^{2}$ Department of Mathematics, Texas A\&M University-Kingsville, Kingsville, Texas 78363, USA.

\section{Acknowledgements}

This research is supported by the National Natural Science Foundation of China (No. 11101385) and by the Fundamental Research Funds for the Central Universities.

Received: 9 January 2014 Accepted: 7 April 2014 Published: 07 May 2014

\section{References}

1. Graef, JR, Kong, L, Mihós, FM: Higher order $\phi$-Laplacian BVP with generalized Sturm-Liouville boundary conditions. Differ. Equ. Dyn. Syst. 18(4), 373-383 (2010)

2. Graef, JR, Kong, L, Mihós, FM, Fialho, J: On the lower and upper solution method for higher order functional boundary value problems. Appl. Anal. Discrete Math. 5, 133-146 (2011)

3. Grossinho, MR, Minhós, FM: Existence result for some third order separated boundary value problems. Nonlinear Anal. 47, 2407-2418 (2001)

4. Bai, Z: Positive solutions of some nonlocal fourth-order boundary value problem. Appl. Math. Comput. 215, 4191-4197 (2010)

5. Du, Z, Liu, W, Lin, X: Multiple solutions to a three-point boundary value problem for higher-order ordinary differential equations. J. Math. Anal. Appl. 335, 1207-1218 (2007)

6. Agarwal, RP, O'Regan, D: Infinite Interval Problems for Differential, Difference and Integral Equations. Kluwer Academic, Dordrecht (2001)

7. Countryman, M, Kannan, R: Nonlinear boundary value problems on semi-infinite intervals. Comput. Math. Appl. 28, 59-75 (1994)

8. Philos, CG: A boundary value problem on the half-line for higher-order nonlinear differential equations. Proc. R. Soc. Edinb. 139A, 1017-1035 (2009)

9. Umamahesearam, S, Venkata Rama, M: Multipoint focal boundary value problems on infinite interval. J. Appl. Math. Stoch. Anal. 5, 283-290 (1992)

10. Agarwal, RP, O'Regan, D: Nonlinear boundary value problems on the semi-infinite interval: an upper and lower solution approach. Mathematika 49, 129-140 (2002)

11. Eloe, PW, Kaufmann, ER, Tisdell, CC: Multiple solutions of a boundary value problem on an unbounded domain. Dyn. Syst. Appl. 15(1), 53-63 (2006)

12. Lian, H, Wang, P, Ge, W: Unbounded upper and lower solutions method for Sturm-Liouville boundary value problem on infinite intervals. Nonlinear Anal. 70, 2627-2633 (2009)

13. Lian, $\mathrm{H}$, Zhao, J: Existence of unbounded solutions for a third-order boundary value problem on infinite intervals. Discrete Dyn. Nat. Soc. (2012). doi:10.1155/2012/357697

14. Yan, B, O'Regan, D, Agarwal, RP: Unbounded solutions for singular boundary value problems on the semi-infinite interval: upper and lower solutions and multiplicity. J. Comput. Appl. Math. 197, 365-386 (2006)

15. Zhao, Y, Chen, H, Xu, C: Existence of multiple solutions for three-point boundary-value problems on infinite intervals in Banach spaces. Electron. J. Differ. Equ. 44, 1-11 (2012)

16. Ehme, J, Eloe, PW, Henderson, J: Upper and lower solution methods for fully nonlinear boundary value problems. J. Differ. Equ. 180, 51-64 (2002)

17. Agarwal, RP, O'Regan, D: Infinite interval problems modeling phenomena which arise in the theory of plasma and electrical potential theory. Stud. Appl. Math. 111, 339-358 (2003)

18. Baxley, JV: Existence and uniqueness for nonlinear boundary value problems on infinite interval. J. Math. Anal. Appl. $147,122-133$ (1990)

19. Chen, S, Zhang, Y. Singular boundary value problems on a half-line. J. Math. Anal. Appl. 195, 449-468 (1995)

20. Gross, OA: The boundary value problem on an infinite interval, existence, uniqueness and asymptotic behavior of bounded solutions to a class of nonlinear second order differential equations. J. Math. Anal. Appl. 7, 100-109 (1963)

21. Jiang, D, Agarwal, RP: A uniqueness and existence theorem for a singular third-order boundary value problem on $[0,+\infty)$. Appl. Math. Lett. 15, 445-451 (2002)

22. Liu, Y: Boundary value problems for second order differential equations on infinite intervals. Appl. Math. Comput. $135,211-216(2002)$

23. Ma, R: Existence of positive solution for second-order boundary value problems on infinite intervals. Appl. Math. Lett. 16, 33-39 (2003)

24. Bai, C, Li, C: Unbounded upper and lower solution method for third-order boundary-value problems on the half-line. Electron. J. Differ. Equ. 119, 1-12 (2009) 
10.1186/1687-2770-2014-100

Cite this article as: Lian et al.: Upper and lower solution method for $n$ th-order BVPs on an infinite interval. Boundary Value Problems 2014, 2014:100

Submit your manuscript to a SpringerOpen ${ }^{\circ}$ journal and benefit from:

- Convenient online submission

- Rigorous peer review

- Immediate publication on acceptance

- Open access: articles freely available online

- High visibility within the field

- Retaining the copyright to your article

Submit your next manuscript at $\gg$ springeropen.com 